\title{
Generation of Rotationally Symmetric Micro Tools using Ultrashort Laser Pulses
}

\author{
Julian Zettl ${ }^{1}$, Maximilian Klar ${ }^{1}$, Cemal Esen ${ }^{2}$ and Ralf Hellmann ${ }^{1}$ \\ ${ }^{1}$ University of Applied Sciences Aschaffenburg, Applied Laser and Photonics Group, Würzburger \\ Strasse 45, 63743 Aschaffenburg \\ Corresponding author's e-mail: Julian.Zettl@th-ab.de \\ ${ }^{2}$ Ruhr University Bochum, Applied Laser Technologies, Universitätsstrasse 150, 44801 Bochum
}

\begin{abstract}
We report on the fabrication of rotationally symmetrical geometries with ultrashort laser pulses impinging the constant rotating workpiece tangentially. This particular micro machining of geometry is referred to as laser turning, upon which the laser beam is, in general, oriented perpendicularly to the rotational axis and tangentially to the work piece. The target geometry is realized by moving the constantly rotating specimen according to the specified geometry along the focused laser spot. In contrast to mechanical turning, laser turning induces a minimized amount of lateral forces through friction that might deteriorate the geometry. In this study, laser turning is examined for stellite as a function of pulse energy and pulse length in the range between $240 \mathrm{fs}$ and $10 \mathrm{ps}$. Shorter pulse lengths and higher pulse energies increase the achievable ablation rate, while in the pulse length regime of below 1 ps lower roughness is achieved. With a maximum ablation rate of $1.27 \mathrm{~mm}^{3} / \mathrm{min}$ and a minimum roughness of $0.17 \mu \mathrm{m}$, laser turning combines both, fast processing and high surface quality.
\end{abstract}

DOI: $10.2961 /$ jlmn.2020.02.2007

Keywords: ultra-short laser pulses, laser turning, stellite, ablation rate, process efficiency

\section{Introduction}

Lathe turning is, along with, e.g., cutting and drilling, an important and widespread procedure of machining parts of various materials. In this particular process, the work piece rotates around its axis while the lathe chisel is guided alongside the material, thus realizing the geometry of the rotationally symmetric part. However, this mechanical approach holds multiple disadvantages such as wearing of the tool or occurring shear forces, which tend to negatively affect the process. As a consequence, imprecise dimensional accuracy, chipping of brittle materials or even breakage of the part may occur.

Changing the mechanical lathe chisel to a focused ultrashort pulsed laser beam, however, circumvents these disadvantages. This non-contact tool of the highest precision ablates material by irradiating the work piece with optical radiation of high energy density. Combined with the ultrashort pulse length, various materials such as metals, ceramics [1-3] and even transparent materials like fused silica and polymers $[4 ; 5]$ can be machined with minimal thermal effects [6]. The focused laser beam is free of wearing effects and the resulting shear forces are negligible, enabling the processing of miniature and frangible parts.

Many laser-based applications that process rod or tube shaped samples apply radial machining with the laser beam perpendicular to the work piece surface. This setup requires a vast amount of processing know-how to achieve the desired ablation depth, surface quality and to meet the demanded precision. By machining the work piece tangentially, the profiling is achieved by positioning and feeding the laser beam. Therefore, the arrangement is self-limiting in terms of material removal. This favors a high repeat accuracy and stable processing even with material inhomogeneities.
The laser turning process has shown potential regarding part roundness [7] and displayed advantages in the generation of tools in terms of occurring processing forces as well as tool wear [8]. The most common applications of laser turning are dressing, conditioning or profiling of grinding wheels. Thereby, the laser beam is used to restore the abrasive surface of the wheel, either by melting off a surface layer and exposing new grains or by precise material removal to achieve pre-specified flank geometries [9-14]. Furthermore, ultrashort pulsed laser turning can be used to manufacture dental ceramic implants, as shown by Ackerl et al. [15].

While cutting, drilling and structuring of materials with ultrashort laser pulses are prevalent processes [16], the tangential turning operation is still in need of far-reaching exploration and establishment. In this paper, we present a comprehensive study of a tangential laser turning process to generate rotationally symmetric parts from stellite rods using ultrashort laser pulses. Our results highlight that this femtosecond laser lathe process features a promising method for hard to machine materials on a micro scale.

\section{Materials and methods}

\subsection{Laser system}

The experiments were carried out on a four-axis laser micro machining station (WS Multihead, Optec S.A. Belgium). The $\mathrm{X}, \mathrm{Y}$ and $\mathrm{Z}$ axes are used for positioning the laser beam and adjusting the feed rate alongside the work piece while the R-axis implements the rotatory motion as depicted in Fig. 1.

An ultrashort pulse laser system (Carbide, Light Conversion Lithuania) with a maximum average output power of 


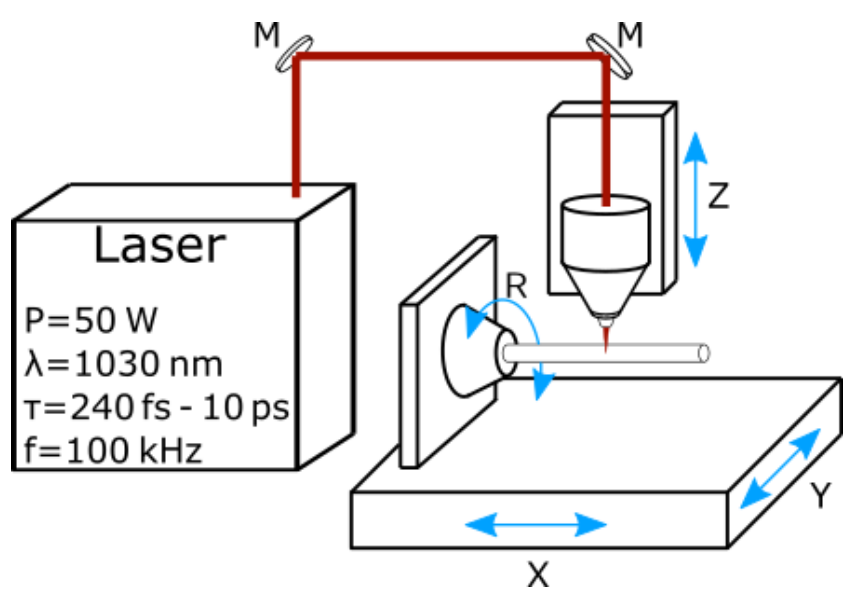

Fig. 1 Setup of laser micro machining station.

$50 \mathrm{~W}$ and a wavelength of $1030 \mathrm{~nm}$ was deployed. At a repetition rate of $100 \mathrm{kHz}$, a maximum pulse energy of $500 \mu \mathrm{J}$ is achieved. The pulse length of the laser can be set between $0.24 \mathrm{ps}-10 \mathrm{ps}$. The laser beam is focused by a cutting head with a focal length of $50 \mathrm{~mm}$, resulting in a calculated focal spot diameter of approximately $18 \mu \mathrm{m}$. A nozzle orifice diameter of $0.5 \mathrm{~mm}$ was chosen and the initial nozzle to work piece distance was set to $1.5 \mathrm{~mm}$. A constant flow of Nitrogen at approximately $15 \mathrm{l} / \mathrm{min}$ was applied as processing gas to firstly guard the lens of the processing head from ablated material and secondly to ensure a continuous removal of debris from the work piece.

\subsection{Materials and process characterization}

Stellite rods (quality K-44 UF, $88 \%$ WC, $12 \%$ Co) with an initial diameter of $1.13 \mathrm{~mm}$ were chosen as test material. With a flexural strength of $>4000 \mathrm{~N} / \mathrm{mm}^{2}$ it is applicable for drilling or shaping of high temperature resistant alloys, cast iron, stainless steels or titanium alloys. The rods are processed tangentially while rotating around their axis at a speed of $500 \mathrm{rpm}$ as depicted in Fig. 2. The focused laser beam is guided parallel to the rotation axis of the work piece. The distance between the outer circumference and the laser tool path is referred to as offset. Depending on the initial work piece diameter, the offset defines, together with the applied pulse energy and the laser spot radius, the ablation depth in the material and thus concludes the final contour of the generated geometry. Therefore, the maximum ablation depth of this process is limited to the chosen offset plus the laser spot radius. Hence, this process is self-limiting in such a way that the chosen offset defines the nominal applicable ablation area. Throughout the process, the laser focal plane

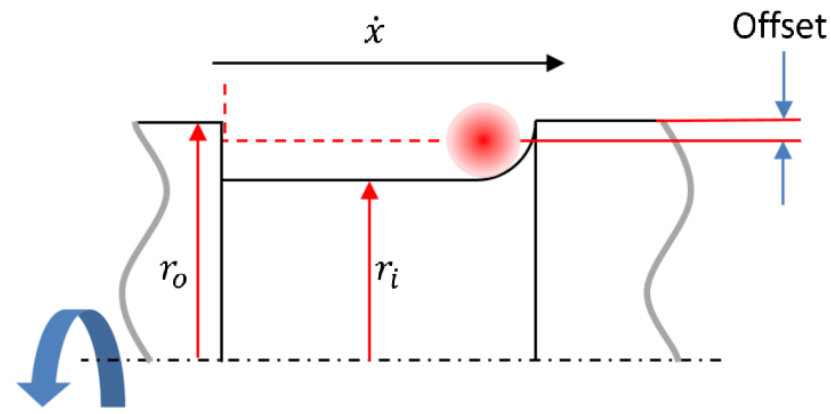

Fig. 2 Schematic illustration of the realized geometry for calculating the ablation rate and ablation efficiency. is kept on the same level as the rotation axes of the work piece.

The correlation of the rotation speed $R$ of the work piece, the feed rate $\dot{x}$ of the laser alongside the rotation axes and the laser spot diameter $d_{s}$ affect the result of the laser turning process. Exceeding the critical feed rate $\dot{x}_{\text {crit }}=R \cdot d_{s}$ causes a separation of the processing paths on the cylindrical surface of the work piece as shown in Fig. 3, potentially and inadvertently creating a thread.

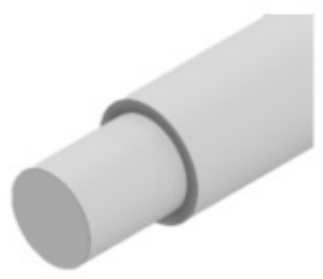

$\dot{\mathrm{x}} \leq \mathrm{R} \cdot \mathrm{d}_{\mathrm{s}}$

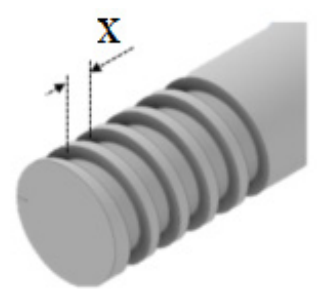

$\dot{\mathrm{x}}>\mathrm{R} \cdot \mathrm{d}_{\mathrm{s}}$
Fig. 3 Critical feed rate for material removal.

For determining the ablation rate, the initial and the final diameter are measured with an optical microscope. The ablation rate $\dot{V}$ is calculated according to

$$
\dot{V}=\left(\left(r_{o}^{2}-r_{i}^{2}\right) \cdot \pi\right) \cdot \dot{x}
$$

with $r_{i}$ representing the inner (final) radius, $r_{o}$ the outer radius and $\dot{x}$ the relative speed of the laser alongside to the rotation axis (cf. Fig. 2).

The arithmetic average roughness $R_{a}$ was measured by a laser scanning microscope (Keyence VK - X200, Keyence Corp.).

\section{Results and discussion}

3.1 Influence of pulse length on ablation rate and efficiency

To characterize the effect of the laser pulse length onto the laser turning process, a series of studies are conducted to investigate its impact on the ablation rate at increasing pulse energies. The initial work piece diameter is $1.13 \mathrm{~mm}$, the offset is set to $100 \mu \mathrm{m}$ and the feed rate $0.05 \mathrm{~mm} / \mathrm{s}$. The ablation rates for pulse lengths of $0.24 \mathrm{ps}, 1 \mathrm{ps}$ and $10 \mathrm{ps}$ are summarized in Fig. 4.

The presented results are single measurement values. However, the results are based upon several preliminary

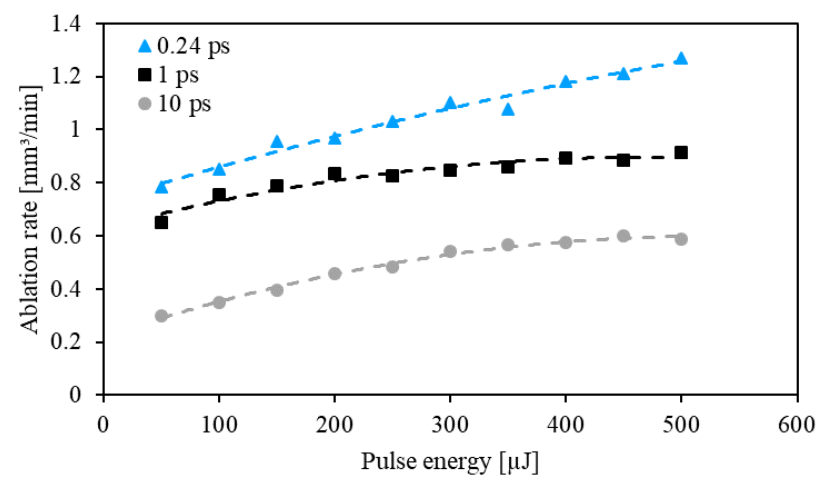

Fig. 4 Ablation rate over pulse energy for different pulse lengths. Dashed lines: Guide to the eye. 
tests, which substantiate their credibility as well as their replicability.

Apparently, for the laser turning process a shorter pulse length is beneficial in terms of achievable ablation rate, independent of pulse energy, which is in the agreement with the results of Neuenschwander et al. [17].

Independent of the pulse length, the ablation rate increases with pulse energy. While the slope of the $1 \mathrm{ps}$ and $10 \mathrm{ps}$ curve seem to saturate at pulse energies of $400 \mu \mathrm{J}$ and $450 \mu \mathrm{J}$, respectively, the pulse energy dependence of the ablation rate for a pulse length of 0.24 ps appears to reach its maximum for higher fluencies beyond $500 \mu \mathrm{J}$. The highest removal rate of $1.27 \mathrm{~mm}^{3} / \mathrm{min}$ achieved in this study is reached at a pulse length of $0.24 \mathrm{ps}$ and a pulse energy of $500 \mu \mathrm{J}$.

Fig. 5 depicts the ablation efficiency, defined by the ratio of ablation rate and the applied laser power. A maximum ablation efficiency of $0.16\left(\mathrm{~mm}^{3} / \mathrm{min}\right) / \mathrm{W}$ can be reached with a pulse length of $0.24 \mathrm{ps}$ and an applied pulse energy of $50 \mu \mathrm{J}$. A shorter pulse length proves to be beneficial for increasing the ablation efficiency. Again, this behavior is in accordance to previous studies [17]. When the pulse energy is increased, a decrease in efficiency can be observed for each tested pulse length.

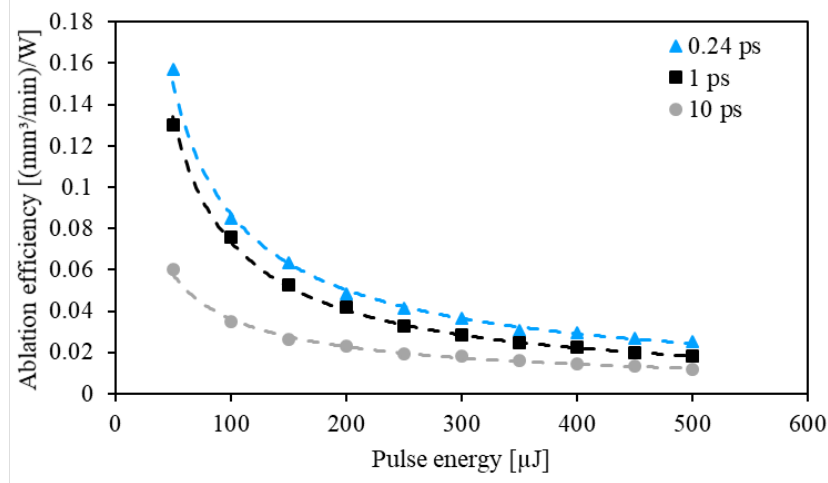

Fig. 5 Ablation efficiency versus pulse energy for different pulse lengths. Dashed lines: Guide to the eye.

The relatively low ablation rate and ablation efficiency compared to conventional, perpendicular laser machining with ultrashort laser pulses is contributed to the high angular beam incidence during processing. The reflectivity of the surface can be described by the Fresnel-Equations [18] for orthogonal and parallel polarized waves as:

$$
\begin{aligned}
& r_{x}=\frac{n_{1} \cos \theta_{1}-n_{2} \cos \theta_{2}}{n_{1} \cos \theta_{1}+n_{2} \cos \theta_{2}} \\
& r_{y}=\frac{n_{2} \cos \theta_{1}-n_{1} \cos \theta_{2}}{n_{2} \cos \theta_{1}+n_{1} \cos \theta_{2}}
\end{aligned}
$$

The resulting absorptivity is given by $A=1-R$. Fig. 6 illustrates the angle dependent absorption for perpendicular and parallel polarization for metal based on equation (2) and (3) with the refractive indices $n_{1}=1$ (air) and $n_{2}=3.08$ (Tungsten, Ordal et al. [19]).

With the initial work piece diameter of $1.13 \mathrm{~mm}$ and an offset of $100 \mu \mathrm{m}$, the incident beam angle calculates to approximately $55^{\circ}$. With an undefined orientation of the linear polarized incident laser beam, the absorbed laser power can decrease significantly. Parallel orientation of the polarization linked with an angularly optimized offset, on the other hand, can drastically improve the absorption and therefore increase the ablation rate and the overall efficiency of the laser turning process.

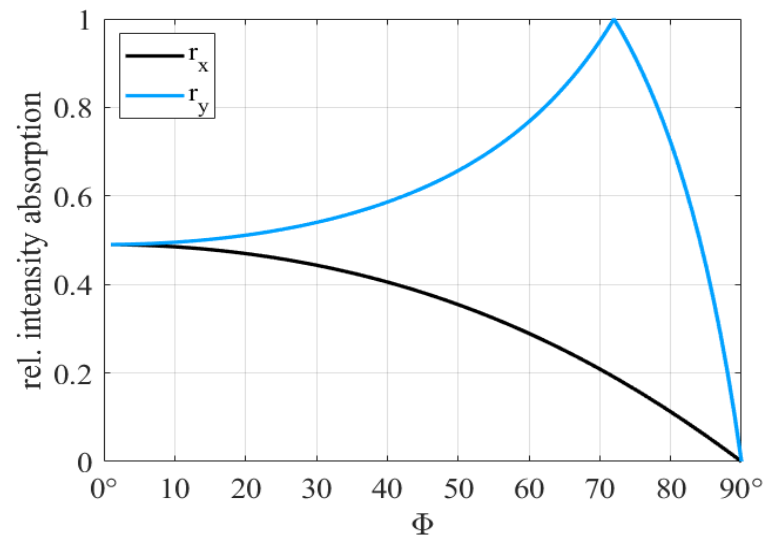

Fig. 6 Angle dependent absorption for perpendicular and parallel polarization in Tungsten.

\subsection{Influence of pulse length on surface topography}

To survey the influence of the applied pulse length onto the forming surface, a series of roughness measurements after the laser turning process was performed. Pulse lengths of $0.24 \mathrm{ps}, 1 \mathrm{ps}$ and $10 \mathrm{ps}$ and pulse energies between $50 \mu \mathrm{J}$ and $500 \mu \mathrm{J}$ were applied, whereat the arithmetic mean deviation of the roughness profile was measured. The measurements were taken after two consecutive passes of each parameter. The resulting values are depicted in Fig. 7.

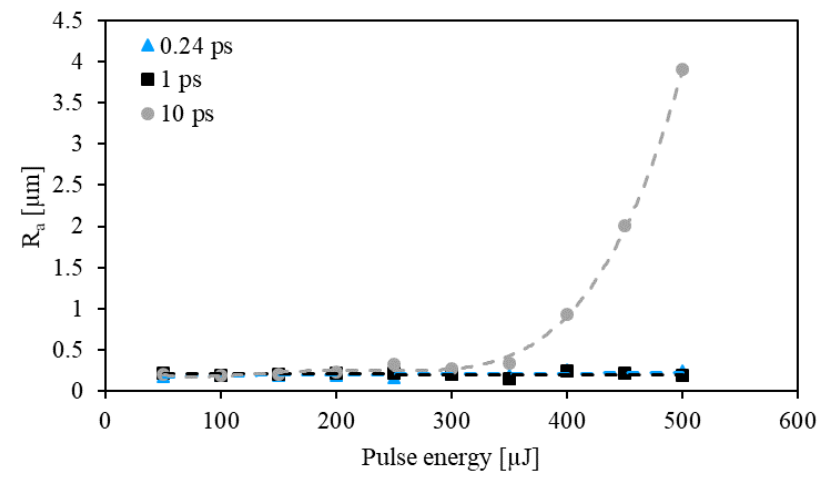

Fig. 7 Arithmetic mean deviation of the roughness profile versus pulse energy. Dashed lines: Guide to the eye.

The graph indicates that pulse lengths of $1 \mathrm{ps}$ and less result in similar surface qualities with a roughness $R_{a}$ of approximately $0.21 \mu \mathrm{m}$ for all examined pulse energies. The lowest roughness of $0.17 \mu \mathrm{m}$ is measured for a pulse length of $0.24 \mathrm{ps}$ and a pulse energy of $250 \mu \mathrm{J}$. At a pulse length of $10 \mathrm{ps}$, however, the roughness increases significantly for pulse energies of $350 \mu \mathrm{J}$ and above, suggesting an increase in melting effects. The microscope image of the study with a pulse length of $10 \mathrm{ps}$ is shown in Fig. 8. Starting at $50 \mu \mathrm{J}$ (left), a gradual increase of the pulse energy by $50 \mu \mathrm{J}$ per step results in the mentioned melting of the surface at $350 \mu \mathrm{J}$ and above, which is in accordance with Fig. 7. 


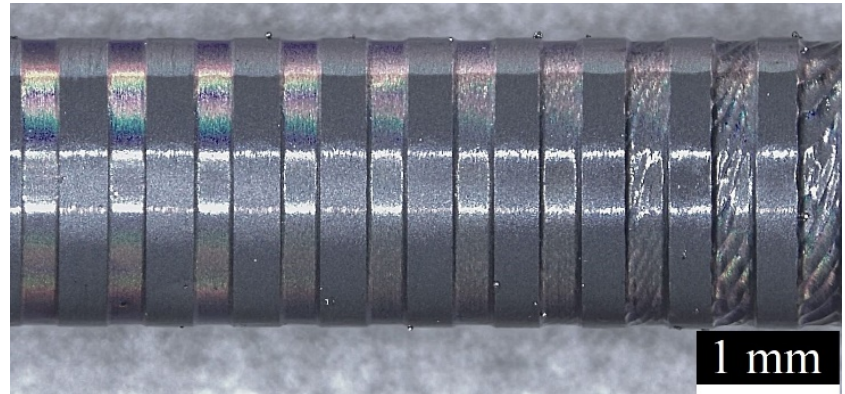

Fig. 8 Surfaces of laser turned K-44UF; Pulse length: 10 ps, Pulse energy: $50-500 \mu \mathrm{J}$ (from left to right).

An inspection by a scanning electron microscope (SEM) reveals that all achieved surfaces show laser induced periodic surfaces (LIPSS) as shown in Fig. 9.

These structures are formed through linear polarized fslaser pulses and are oriented perpendicular to the incident laser polarization [20]. LIPSS occur at a laser fluence close to the material specific ablation threshold fluence and form on various materials [20-23]. Due to the periodicity close to the laser wavelength, the structures can be categorized as low spatial frequency LIPSS (LSFL) [24]. The formation of these structures is in the laser turning process unaffected by the applied pulse energy, as they appear even after high fluence processing (e.g. Fig. 9c).

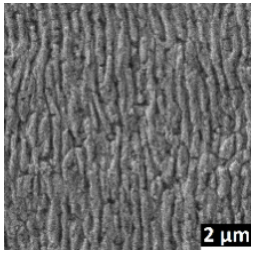

a)

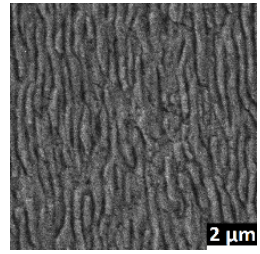

b)

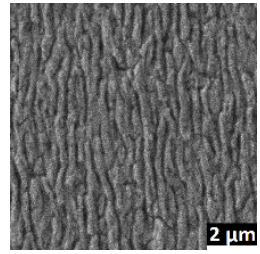

c)
Fig. 9 SEM images of laser turned surfaces with a pulse length of 240 fs. a) $50 \mu \mathrm{J}$; b) $250 \mu \mathrm{J}$; c) $500 \mu \mathrm{J}$.

We assume that because of the high angular beam incidence and the thereby caused high reflectivity of the material, the necessary low fluence regime for the generation of LIPSS is reached at the outer region of the processing laser spot. Hence, the occurrence of LIPSS is currently speculated to be the limitation of achievable roughness in the laser turning process.

\subsection{Tool generation}

The shaping of material in the laser turning process is, except from rotational symmetry and undercuts, freely selectable. Fig. 10 shows an SEM image of an exemplary geometry manufactured through this process.

A variety of shapes such as spheres, arcs and angles of different sizes are possible. Ninety-degree angles, however, are limited by the laser spot radius. Also, when ablating a large amount of material, debris may accumulate on perpendicular features. However, debris can in general be easily removed by sonicating the part. Laser turning can be, much like mechanical lathing, grouped in two processing steps: roughing and smoothing. During the roughing process, the approximate contour is realized while ablating the majority of material. Throughout the smoothing process, the exact outline of the part is realized while only removing little

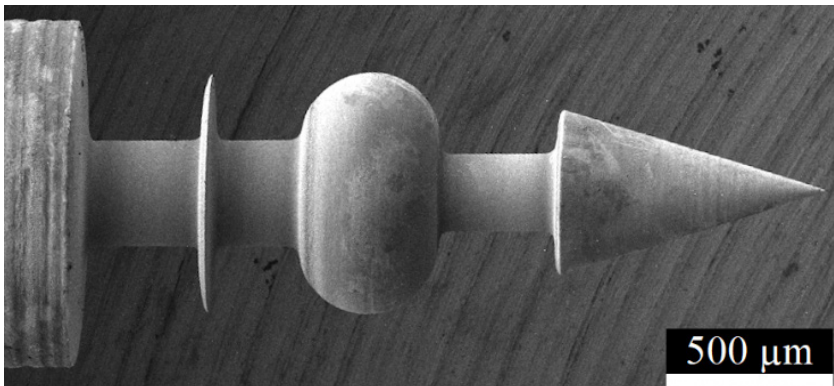

Fig. 10 SEM image of exemplary tool geometry.

amounts of material. In contrast to mechanical lathing, however, both processing steps can be accomplished with the same tool by simply adjusting the feed rate and pulse energy. The overall processing time of the work piece shown in Fig. 10 is about $5 \mathrm{~min}$, highlighting the speed and flexibility of the laser turning process.

\section{Conclusion}

In this report the process of tangential laser turning with ultrashort laser pulses was investigated. Subject of the studies was the influence of pulse length and pulse energy on the feasible ablation rate, efficiency and roughness. Furthermore, a possible influence of the incident laser polarization was discussed. An ablation rate of $1.27 \mathrm{~mm}^{3} / \mathrm{min}$ was achieved and an efficiency of up to $0.16\left(\mathrm{~mm}^{3} / \mathrm{min}\right) / \mathrm{W}$ was demonstrated. The smoothest surface was measured with an arithmetic mean deviation roughness of $0.17 \mu \mathrm{m}$. A shorter pulse length has proven to be beneficial for the laser turning process, both in terms of ablation rate and roughness. Long pulse durations above 1 ps along with high fluencies cause melting effects and negatively affect the processing results. Furthermore, the formation of LIPSS was illustrated and identified as a possible limitation of the achievable roughness. The findings in this report will contribute to foster the development of miniature rotationally symmetric parts and tools.

\section{References}

[1] Y. Liu, R. Zhang, W. Li, J. Wang, X. Yang, L. Cheng and L. Zhang: Int. J. Adv. Manuf. Technol., 96, (2018) 1795.

[2] A. Gruner, J. Schille and U. Loeschner: Phys. Procedia, 83, (2016) 157.

[3] B. Jaeggi, S. Remund, R. Streubel, B. Goecke, S. Bracikowski and B. Neuenschwander: J. Laser Micro/Nanoengin., 12, (2017) 267.

[4] G.-L. Roth, C. Esen and R. Hellmann: J. Laser Appl., 31, (2019) 22603.

[5] S. Schwarz and R. Hellman: J. Laser Micro/Nanoengin., 12, (2017) 76.

[6] S. Mishra and V. Yadava: Opt. Laser Eng., 73, (2015) 89.

[7] S. R. Subramonian, A. Z. Khalim, Hussein, N. I. S, R. Izamshah, M. Amran and M. Hadzley: Journal of Current Engineering Research, 2, (2012) 98.

[8] M. Warhanek, C. Walter, M. Hirschi, J. Boos, J. F. Bucourt and K. Wegener: J. Manuf. Process., 23, (2016) 157. 
[9] A. H. A. Lutey, A. Fortunato, F. Zanini and S. Carmignato: Lasers Manuf. Mater. Process., 3, (2016) 158.

[10] M. Mukhopadhyay and P. K. Kundu: Int. J. Mechatron. Manuf. Syst., 11, (2018) 167.

[11] K. Wegener, H.-W. Hoffmeister, B. Karpuschewski, F. Kuster, W.-C. Hahmann and M. Rabiey: CIRP Ann. Manuf. Technol., 60, (2011) 757.

[12] N. Ackerl, M. Warhanek, J. Gysel and K. Wegener: Mater. Des., 189, (2019) 108530.

[13] G. Chen, S. Cai and C. Zhou: Diam. Relat. Mater., 60, (2015) 99.

[14] C. Genyu, W. Yi, P. YanBo and W. Yanyi: Int. J. Adv. Manuf. Technol., 104, (2019) 4077.

[15] N. Ackerl, M. Warhanek, J. Gysel and K. Wegener: J. Eur. Ceram. Soc., 39, (2019) 1635.

[16] J. Bliedtner, C. Schindler, M. Seiler, S. Wächter, M. Friedrich and J. Giesecke: Laser Tech. J., 5, (2016) 46.

[17] B. Neuenschwander, B. Jaeggi, M. Schmid, V. Rouffiange and P.-E. Martin: Proceedings of SPIE, (2012), \#8243-07.
[18] B. E. A. Saleh, M. C. Teich: "Fundamentals of Photonics" ed. by Goodman, J. W. (John Wiley \& Sons, Inc., New York 1991) p. 205.

[19] M. A. Ordal, R. J. Bell, R. W. Alexander, L. A. Newquist and M. R. Querry: Appl. Opt., 27, (1988) 1203.

[20] J. Bonse, M. Munz and H. Sturm: Appl. Surf. Sci., 97, (2005) 13538.

[21] S. Gräf and F. A. Müller: Appl. Surf. Sci., 331, (2015) 150 .

[22] A. Y. Vorobyev, V. S. Makin and C. Guo: J. Appl. Phys., 101, (2007) 34903.

[23] A. Borowiec and H. K. Haugen: Appl. Phys. Lett., 82, (2003) 4462.

[24] J. Bonse, S. Hohm, S. V. Kirner, A. Rosenfeld and J. Kruger: IEEE J. Select. Topics Quantum Electron., 23, (2017) 9000615.

(Received: June 26, 2020, Accepted: August 25, 2020) 